\title{
Uso de la clasificación de Robson en las cesáreas practicadas en el Hospital Juárez de México durante los años 2012-2016
}

\author{
Rufina Reyes-Morales ${ }^{1}$, Víctor Manuel Vargas-Hernández ${ }^{1}$ y José María Tovar-Rodríguez ${ }^{*}$ \\ ${ }^{1}$ División de Ginecología y Obstetricia; ${ }^{2}$ División de Investigación. Hospital Juárez de México, Ciudad de México, México
}

\begin{abstract}
RESUMEN
Antecedentes: La clasificación de Robson establece grupos de embarazadas con características específicas que identifican al grupo con mayor prevalencia de cesáreas y así poder tomar medidas para disminuir la tasa de cesáreas. Robson propuso un sistema de 10 grupos, que clasifica a todas las mujeres de acuerdo con 5 características obstétricas: paridad, comienzo del trabajo de parto, edad gestacional, presentación fetal y cantidad de fetos. Objetivo: Identificar el grupo de mujeres en quienes se realiza el mayor número de cesáreas en el Hospital Juárez de México. Métodos: Se revisaron los expedientes de la muestra de pacientes que se atendieron por parto o cesárea a partir de las 28 semanas en el periodo de 2012 a 2016. Resultados: La muestra anual fue de 378 nacimientos. La tasa de cesáreas en los 5 años fue del 47.7\%. El grupo 5 fue el que más aportó a la tasa de cesáreas (31.8\%), seguido del grupo 1 (23.9\%) y en tercer lugar el grupo 10 (15.7\%). La indicación más frecuente fue cesárea iterativa, seguida de falta de progresión de trabajo de parto. Conclusión: Para reducir la tasa de cesáreas es necesario evitar la primera cesárea y favorecer el parto después de cesárea.
\end{abstract}

Palabras clave: Cesárea. Clasificación Robson. Parto. Embarazo.

\section{ABSTRACT}

Background: Robson classification establishes groups of pregnant women with specific characteristics, which identifies the group with the highest prevalence of cesarean sections allowing to take measures to reduce the rate of caesarean section. He proposed a system of 10 groups, which classifies all women according to 5 obstetric characteristics: parity, labor initiation, gestational age, fetal presentation, and number of fetuses. Objective: To identify the group of women in whom the highest number of caesarean sections are performed at the Hospital Juárez de México. Methods: We reviewed the records of patients who were attended by delivery or caesarean section from 28 weeks onwards in the period of 2012 to 2016 . Results: The annual sample was 378 births. The cesarean section rate for the 5 years was $47.7 \%$. Group 5 was the one which contributed the most to the cesarean section rate (31.8\%), followed

\section{Correspondencia:}

*José María Tovar-Rodríguez

E-mail: gacetajuarez@gmail.com
Date of reception:18-09-2018

Date of acceptance: 11-03-2019

DOI: 10.24875/HMCM.19000157 
by group $1(23.9 \%)$ and group 10 (15.7\%). The most frequent indication was an iterative cesarean, followed by a lack of progression of labor. Conclusion: To reduce the rate of caesarean sections it is necessary to avoid the first caesarean section and favor delivery after a caesarean section. (Hosp Med Clin Manag. 2019;12:13-21)

Corresponding author: José María Tovar-Rodríguez, gacetajuarez@gmail.com

Key words: Cesarean section. Robson classification. Childbirth. Pregnancy.

\section{INTRODUCCIÓN}

El parto por cesárea es la cirugía mayor más común realizada en México y en el resto del mundo. A nivel mundial se realizan aproximadamente 18.5 millones de cesáreas de las cuales la mitad no están justificadas. La tasa de cesáreas en el Hospital Juárez de México ha ido en incremento; en el año 2001 se registró una tasa de cesáreas del $31.7 \%$ y en el 2014 de 50.7\% (datos no publicados), por lo que es necesario implementar una estrategia para disminuir la tasa de cesáreas en nuestro hospital.

Desde 1985, los profesionales de la salud en todo el mundo han considerado que la tasa ideal de cesáreas debe mantenerse entre el 10 y el $15 \%{ }^{1}$. Estas cifras han sido muy criticadas y debatidas; sin embargo, la cesárea continúa presentando un aumento preocupante en todo el mundo. La operación de cesárea debe prevenir la mortalidad y la morbilidad maternoperinatal. Hasta el momento no se ha demostrado mayor beneficio en mujeres o en el recién nacido que en las mujeres en las que no se requiere la interrupción del embarazo por vía abdominal ${ }^{1,2}$. Aún no se ha determinado la causa de este incremento en la tasa de cesárea, pero forma parte de un complejo multifactorial que implica a los sistemas de salud, los proveedores de cuidado de la salud, las mujeres, las sociedades e incluso la moda. Hasta la fecha, las intervenciones para reducir el número de cesáreas no han sido eficaces².

Secundario a esto, la Organización Mundial de la Salud (OMS), en octubre de 2014, recomendó una tasa de cesáreas del 10-15\%; esto se basó en las tasas de parto por cesárea en los países que tenían las tasas de mortalidad materna y neonatal más bajas en el mundo 3 . La OMS concluye que las tasas superiores al 10-15\% no se asocian con reducciones en las tasas de mortalidad materna y neonatal².

Se han hecho estudios relacionando la vía de nacimiento con la mortalidad perinatal, demostrándose que los países con las tasas de cesárea por debajo del 15\% presentaron asociación negativa con la mortalidad materna. Sin embargo, para los países con la tasa de cesárea más altas (por encima del 15\%), estas correlacionaron con mayor mortalidad materna ${ }^{3}$.

Al igual que con cualquier cirugía, la cesárea está asociada a riesgos a corto y largo plazo, principalmente cuando se realizan en condiciones inadecuadas de infraestructura y con personal no capacitado. Por otra parte, la realización inadecuada e inoportuna de una cesárea puede dar como resultado asfixia perinatal, muerte fetal, rotura uterina o fístulas obstétricas. Por lo tanto, las cesáreas deben realizarse cuando realmente sean necesarias por indicaciones médicas y proporcionarlas a todas las mujeres que lo necesiten?2.

Existen factores no modificables para la realización de una cesárea, tales como una mala presentación fetal, frecuencia cardíaca fetal desalentadora antes o durante el trabajo de parto, macrosomía y malformaciones fetales. Factores obstétricos potencialmente modificables incluyen inducción fallida, detención del trabajo de parto, gestaciones múltiples, preeclampsia, distocia de hombros, miomectomía previa, antecedente de desgarro de tercer o cuarto grado, placentación marginal y de inserción baja. Los factores maternos modificables para evitar una primera cesárea incluyen: obesidad, infección, enfermedades cardiovasculares, pelvis inadecuadas y la solicitud de la paciente sin indicaciones maternas, obstétricas o fetales. La preocupación por los riesgos de un parto vaginal y menos preocupación por los riesgos de una cesárea puede conducir a la decisión de realizar una cesárea ${ }^{4-5}$. El enfoque más efectivo para reducir la morbilidad general está encaminado a disminuir la primera cesárea ${ }^{5}$.

Los médicos deben informar detalladamente a las pacientes y concienciarlas de las posibles complicaciones derivadas de cirugías repetidas después de la primera cesárea. El ingreso de las pacientes antes de 
tiempo, cuando el trabajo de parto está en fase inicial o sin indicación médica, favorece estancias prolongadas en el área de tococirugía, lo que puede causar fatiga de la paciente o la decisión del médico de realizar una cesárea, la carga de trabajo de muchos trabajadores de salud también afecta la toma de decisiones. La corriente climática médico-legal ha hecho que la espera de un parto vaginal sea menos atractiva para muchos médicos en trabajos de parto que están progresando bien. Las expectativas de las pacientes, el clima médico-legal y los patrones de la práctica relacionada con manejo durante el parto debe dirigirse a reducir la tasa de la primera cesárea. La educación en relación con el curso normal del trabajo de parto y las implicaciones de la primera cesárea pueden permitir a las mujeres y sus médicos evitar prácticas que aumentan la práctica de cesáreas innecesarias 5 .

Se ha observado que la razón de momios (OR) para la mortalidad neonatal de cesárea primaria indicada sin riesgo era 2.02 ((intervalo de confianza [IC] 95\%: 1.602.55) en comparación con el parto vaginal. Por lo tanto, la cesárea debe utilizarse con precaución ${ }^{3}$.

La clasificación de las cesáreas siempre ha sido problemática debido a la falta de definiciones uniformes y ha dado lugar a una mala reproducibilidad y comparaciones insatisfactorias. En el año 2001, el Dr. Michael Robson propuso un sistema de 10 grupos que clasifica a todas las mujeres admitidas a la sala de parto de acuerdo con 5 características obstétricas ${ }^{2,6}$ : paridad (nulípara, multípara con y sin cesárea previa), comienzo del trabajo de parto (espontáneo, inducido o cesárea antes del comienzo del trabajo de parto), edad gestacional (parto prematuro o a término), presentación fetal (cefálica o podálica, transversa) y cantidad de fetos (único o múltiple) ${ }^{1}$. Esta clasificación permite la estratificación de las tasas de cesárea en grupos más uniformes de mujeres y también la evaluación de las tasas de cesárea en relación con otros procesos y resultados perinatales $^{2}$.

La cesárea está asociada a riesgos a corto y a largo plazo que pueden perdurar muchos años después de la intervención y afectar a la salud de la mujer y del neonato, así como a cualquier embarazo futuro. El parto por cesárea electiva sin indicación médica también puede contribuir a un cierto aumento en el riesgo de muerte de la madre. El riesgo de muerte causado

\section{Tabla 1. Clasificación de Robson}

\section{Grupos de mujeres incluidas}

1. Nulíparas con un feto único en presentación cefálica, de 37 semanas 0 más de embarazo, que han iniciado el parto de forma espontánea

2. Nulíparas con un feto único en presentación cefálica, de 37 semanas o más de embarazo, que han sido sometidas a inducción 0 a cesárea antes del inicio del parto

3. Multíparas sin cesárea previa, con un feto único en presentación cefálica, de 37 semanas o más de embarazo, que han iniciado el parto de forma espontánea

4. Multíparas sin cesárea previa, con un feto en presentación cefálica, de 37 semanas 0 más de embarazo, que han sido sometidas a inducción 0 a cesárea antes del inicio del parto

5. Multíparas con al menos una cesárea previa, con un feto único en presentación cefálica, de 37 semanas o más de embarazo

6. Nulíparas con un feto único en presentación podálica

7. Multíparas con un feto único en presentación podálica, incluyendo aquellas con cesárea previa

8. Todas las mujeres con embarazo múltiple, incluyendo aquellas con cesárea previa

9. Todas las mujeres con feto único en posición transversa u oblicua, incluyendo aquellas con cesárea previa

10. Todas las mujeres con un feto único en presentación cefálica de menos de 37 semanas de embarazo, incluyendo aquellas con cesárea previa

por la operación de cesárea es de aproximadamente de 2 por 100,000 cesáreas, comparado con 0.2 por 100,000 muertes causadas por nacimiento vaginal. Por lo tanto, es necesario hacer un diagnóstico situacional de la tasa de cesárea en el hospital e identificar los grupos vulnerables para plantear una estrategia para disminuir la tasa de cesáreas ${ }^{7}$. La clasificación de Robson permitió identificar que los grupos 1 y 3 presentaban incrementada la OR en número de óbitos y mortalidad materna por cesárea en comparación con el parto vaginal ${ }^{8}$. El objetivo del trabajo es identificar el grupo de mujeres en quienes se realiza el mayor número de cesáreas en el Hospital Juárez de México y categorizarlas de acuerdo al sistema de clasificación de Robson.

\section{MÉTODOS}

Se realizó un estudio retrospectivo, transversal, observacional, descriptivo a 5 años de todos los nacimientos por vía vaginal o cesárea, de 28 semanas de gestación en adelante, ocurridos en el Hospital Juárez de México en el periodo del 1 enero 2012 al 31 de diciembre 2016, agrupando a todas las pacientes dentro de los $10 \mathrm{gru}-$ pos de la clasificación de Robson (Tabla 1). Se 
capturaron las variables de edad, cesárea, aborto, presentación y situación fetal, semanas de gestación, presencia o no de trabajo de parto, número de fetos, antecedentes de cirugías uterinas previas e indicación de la cesárea. La tasa de cesáreas fue calculada en cada grupo. La información de la vía de terminación del embarazo fue obtenida de los expedientes de las pacientes.

Se obtuvo la información necesaria de cada mujer embarazada para incluirla en el sistema de 10 grupos que clasifica a todas las mujeres admitidas a la sala de parto de acuerdo con 5 características obstétricas: paridad (nulípara, multípara con y sin cesárea previa), comienzo del trabajo de parto (espontáneo, inducido o cesárea antes del comienzo del trabajo de parto), edad gestacional (parto prematuro o a término), presentación fetal (cefálica o podálica, transversa) y cantidad de fetos (único o múltiple) ${ }^{10-13}$.

\section{Análisis estadístico ${ }^{14}$}

Se calculó la relación entre las pacientes pertenecientes a cada grupo de Robson con respecto al total de la población atendida ([n. ${ }^{\circ}$ de pacientes atendidas pertenecientes a cada grupo/n. ${ }^{\circ}$ total de pacientes atendidas, por vía vaginal y cesárea] x 100), lo cual representa el tamaño relativo de cada uno de los grupos. Se calculó la proporción específica de cesáreas realizadas dentro de cada grupo ([n. ${ }^{\circ}$ de cesáreas realizadas a mujeres pertenecientes a cada grupo/n. ${ }^{\circ}$ de nacimientos vaginales y por cesárea pertenecientes a ese mismo grupo] x 100). Para cuantificar la contribución hecha por cada grupo al porcentaje general de partos se dividió el número de cesáreas en el grupo por el total de partos atendidos en el periodo ( $\left[n{ }^{\circ}\right.$ de total de cesáreas realizadas $/ n{ }^{\circ}$ total de nacimientos, vaginales y por cesárea] x 100).

\section{RESULTADOS}

Durante el periodo estudiado en el Hospital Juárez de México se atendieron un total de 9,802 partos, de donde se hace un cálculo de tamaño de muestra anual de 378 nacimientos (total 1,893 casos) con los siguientes resultados. El promedio de edad de las pacientes fue 24.7 años y el promedio de la edad gestacional fue 38.4 semanas. Las características seleccionadas de las pacientes y sus embarazos se muestran en la tabla 2. Las
Tabla 2. Características clínicas de la población atendida en el Hospital Juárez de México en el periodo 2012-2016

\begin{tabular}{lc}
\hline Características & Valor \\
\hline Edad (años) & 24.6 años \\
Paridad (primigestas) & $41.9 \%$ \\
Numero de fetos, feto único & $98.7 \%$ \\
Edad gestacional (semanas promedio) & 38.4 semanas \\
$\quad$ <28 semanas & $0.9 \%$ \\
$28-37$ semanas & $14.1 \%$ \\
$>37$ semanas & $85.0 \%$ \\
Cesárea previa & $67 \%$ \\
Presentación cefálica & $97.2 \%$ \\
\hline
\end{tabular}

características de la población a lo largo del periodo estudiado no mostraron cambios respecto a la edad, paridad, edad gestacional y proporción de feto único y múltiple.

En la tabla 3 se muestra la distribución de la población respecto a los grupos de Robson, la distribución de partos sobre cesáreas, el porcentaje de cesáreas y la contribución de cada grupo a la tasa de cesáreas. Los grupos 1, 3 y 5 fueron los más numerosos, representando el $81 \%$ de la población. En la figura 1 se representa el grupo de Robson más numeroso, que fue el de primigestas, con embarazo de término, producto en presentación cefálica y trabajo de parto espontáneo (grupo 1), representando 33\% de la población atendida. Siguió el grupo 3, mujeres multigestas con embarazo de término, producto en presentación cefálica y trabajo de parto espontáneo. Las mujeres con cesáreas previas con embarazo a término, con feto único, representaron el tercer grupo más numeroso en nuestra población obstétrica y constituyen el grupo que más aportó al porcentaje de cesáreas. El $11.5 \%$ de las pacientes cursaron con embarazo pretérmino, de las cuales al 65.4\% se les practicó una cesárea. La tasa promedio de cesáreas en los 5 años fue del 47.7\%. El grupo que más aportó a la tasa de cesáreas fue el de las multigestas, con un $60.5 \%$.

De manera individual, el grupo que más aportó a la tasa de cesáreas fue el de mujeres multíparas con antecedente de cicatriz uterina previa (grupo 5), con un 31.8\%, seguido del grupo de mujeres primigestas con embarazo de 37 semanas o más con trabajo de parto espontáneo (grupo 1), con un 23.9\%, y en tercer lugar las mujeres con embarazo menor de 37 semanas (grupo 10), con un $15.7 \%$. 
Tabla 3. Distribución y tasa de cesáreas por grupos de Robson en el Hospital Juárez de México en el periodo 2012-2016

\begin{tabular}{lrrrrr}
\hline Grupo & Tamaño del grupo & \% tamaño grupo & Relación parto/cesárea & $\begin{array}{c}\text { Proporción específica de } \\
\text { cesárea }\end{array}$ & $\begin{array}{c}\text { Contribución de las } \\
\text { cesáreas en el grupo } \\
\text { total }\end{array}$ \\
\hline Grupo 1 & & & & $217 / 630(34.4 \%)$ & $217 / 903(24 \%)$ \\
Grupo 2 & 630 & 33.3 & $413 / 217$ & $78 / 88(88.6 \%)$ & $78 / 903(8.6 \%)$ \\
Grupo 3 & 88 & 4.6 & $10 / 78$ & $63 / 470(13.4 \%)$ & $63 / 903(6.9 \%)$ \\
Grupo 4 & 470 & 24.8 & $407 / 63$ & $25 / 32(78.1 \%)$ & $25 / 903(2.7 \%)$ \\
Grupo 5 & 32 & 1.7 & $7 / 25$ & $288 / 365(79 \%)$ & $288 / 903(31.8 \%)$ \\
Grupo 6 & 365 & 19.3 & $77 / 288$ & $21 / 21(100 \%)$ & $21 / 903(2.3 \%)$ \\
Grupo 7 & 21 & 1.1 & $0 / 21$ & $30 / 30(100 \%)$ & $30 / 903(3.3 \%)$ \\
Grupo 8 & 30 & 1.6 & $0 / 30$ & $19 / 20(95 \%)$ & $19 / 903(2.1 \%)$ \\
Grupo 9 & 20 & 1.1 & $1 / 19$ & $20 / 20(100 \%)$ & $20 / 903(2.2 \%)$ \\
Grupo 10 & 20 & 1.1 & $0 / 20$ & $142 / 217(65.4 \%)$ & $142 / 903(15.7 \%)$ \\
Total & 217 & 11.5 & $75 / 142$ & $903 / 1893(47.7 \%)$ & $903 / 903(100 \%)$ \\
\hline
\end{tabular}

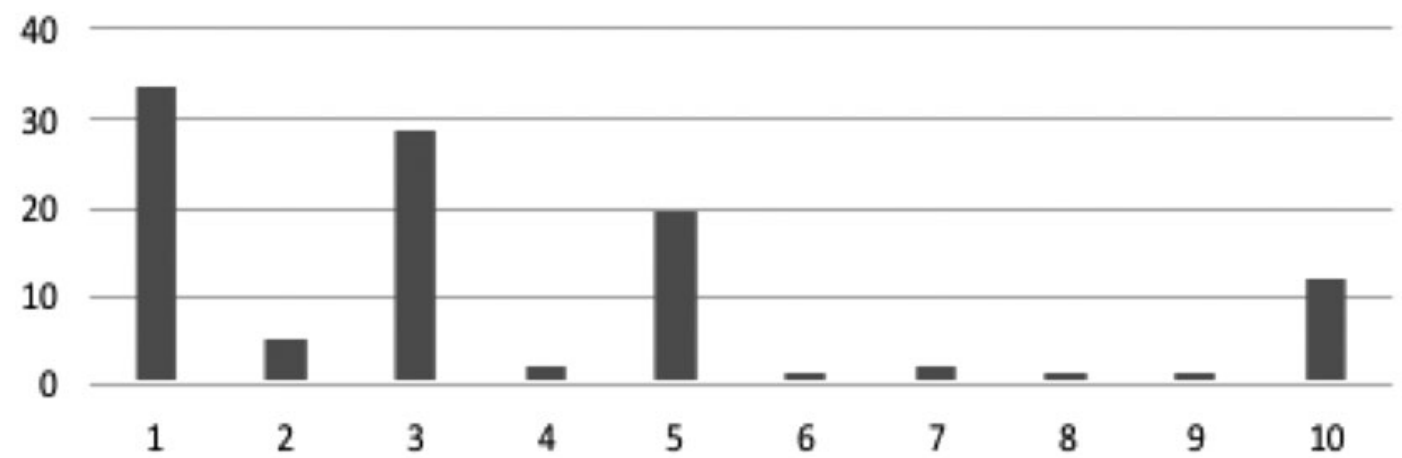

Figura 1. Distribución de las pacientes en los grupos de Robson en el Hospital Juárez de México en el periodo 2012-2016.

La indicación más frecuente de cesárea fue cesárea iterativa. Seguido de falta de progresión de trabajo de parto. En la tabla 4 se muestran las principales indicaciones de cesáreas en nuestro hospital. El grupo que más aportó a la tasa de cesáreas fue el grupo 5, aquellas mujeres con cicatriz uterina previa. Dentro de este grupo la indicación más frecuente fue cesárea iterativa (45\%) y la segunda indicación más frecuente fue cesárea electiva y falta de progresión de trabajo de parto.

El segundo grupo que más aportó a la tasa de cesáreas es el grupo 1, nulíparas con feto único en presentación cefálica de 37 semanas o más que iniciaron el parto en forma espontánea, en el cual la indicación más frecuente de cesárea fue falta de progresión de trabajo de parto, seguido de baja reserva fetal y desproporción cefalopélvica.

El tercer grupo que más aportó a la tasa de cesáreas fue el grupo 10, mujeres con embarazos menores de 37 semanas, con producto único en presentación cefálica.
Dentro de este grupo la principal indicación de cesárea fue trabajo de parto pretérmino, seguido de estados hipertensivos del embarazo y otras patologías. Recientemente la OMS publicó un «Manual de Implementación» y una «Tabla para Reporte de Robson con Interpretación» para el análisis de los resultados obtenidos de la clasificación de Robson de los diferentes grupos analizados ${ }^{15,16}$.

\section{Pasos para asesorar la calidad de los datos}

- Revisar el tamaño del grupo 9 (columna 4); debe ser menor del 1\%. Si es mayor al 1\% es probable que las mujeres con productos en presentación de nalgas $u$ otra presentación hayan sido erróneamente clasificadas como transversas u oblicuas y posteriormente asignadas a este grupo.

- Revisar el porcentaje de cesáreas del grupo 9 (columna 5); debe ser del 100\% por convención. 
Tabla 4. Indicaciones para la operación de cesárea en el Hospital Juárez de México en el periodo 2012-2016

\begin{tabular}{lcc}
\hline Indicación médica & $\mathbf{n}$ & $\%$ \\
\hline Trabajo de parto pretérmino & 45 & 4.9 \\
Falta de progresión de trabajo de parto & 107 & 11.8 \\
Baja reserva fetal & 83 & 9.1 \\
Iterativa/ periodo intergenésico corto & 152 & 16.8 \\
Rotura prematura de membranas/ & 41 & 4.5 \\
oligohidramnios & & \\
Producto pélvico/transverso & 66 & 7.3 \\
Bradicardia fetal & 71 & 7.8 \\
Embarazo múltiple & 20 & 2.2 \\
Cesárea electiva & 55 & 6.0 \\
Desproporción cefalopélvica & 72 & 8.0 \\
Taquicardia fetal & 33 & 3.6 \\
Estados hipertensivos asociados al & 77 & 8.5 \\
embarazo & & \\
Otros & 81 & 9.0 \\
Total & 903 & 100 \\
\hline
\end{tabular}

\section{Pasos para evaluar el tipo de población}

- Revisar el tamaño del grupo 1 + grupo 2 (columna $3)$; representa comúnmente el $35-42 \%$ de la población obstétrica de la mayoría de los hospitales. En nuestra población encontramos que fue del 37.9\%.

- Revisar el tamaño de los grupos 3+4 (columna 3); comúnmente representa cerca del $30 \%$ de mujeres. En nuestro grupo fue del $26.5 \%$.

- Revisar el tamaño del grupo 5 (columna 3); está relacionado a la tasa global de cesárea. El tamaño del grupo 5 es generalmente la mitad de la tasa total de cesárea; está por lo general por debajo del $10 \%$ y en nuestro grupo fue del $19.3 \%$. Si el tamaño de este grupo es muy grande, significa que hay una alta tasa de cesárea en los años pasados en ese hospital. En lugares con altas tasas de cesárea el tamaño de este grupo puede ser mayor al $15 \%$.

- Revisar el tamaño de los grupos 6+7 (columna 3); debe ser el 3-4\%. En nuestro grupo fue del 2.7\%. Si el total es más del 4\%, la razón más común es un alto índice de partos pretérmino.

- Revisar el tamaño de grupo 8; debe ser del 1.5-2\%. En nuestro grupo fue del 1.1\%. Si es más alto, el hospital es probablemente terciario o de alta especialidad, o tiene programa de fertilización in vitro. Si es menor, probablemente muchos de los embarazos gemelares son referidos a otras unidades.

- Revisar el tamaño del grupo 10; debe de ser menor al 5\% en la mayoría de los escenarios de riesgo normal. En el Hospital Juárez es del 11.5\%. Si es mayor la tasa de cesárea, esto sugiere que se hacen por restricción en el crecimiento fetal, preeclampsia u otras complicaciones médicas.

- Revisar el tamaño del grupo 1 vs. grupo 2 (dividir el tamaño del grupo 1 entre el tamaño del grupo 2). Generalmente es 2:1 o más alto; en nuestro estudio fue de 7:1. Si la proporción es muy alta se debe revisar la tasa de partos en esta población, lo que indica que no se está induciendo suficiente, o alternativamente el hospital tiene una población de muy bajo riesgo.

- Revisar la proporción del tamaño del grupo 3 vs. grupo 4 (dividir el tamaño de grupo 3 entre el grupo 4); siempre es más alto que la proporción del grupo 1 vs. 2 , en la misma institución. En nuestro estudio fue de 14:1, este es un hallazgo fidedigno y confirma la calidad de los datos y la cultura de organización. Si es menor se debe sospechar pobre calidad de los datos, por error en la clasificación de multíparas que reciben oxitocina para reforzar el trabajo de parto y debieran estar en el grupo 3 y pueden haber sido clasificadas como inducción e incorrectamente agrupadas en el grupo 4.

- Revisar la proporción del tamaño del grupo 6 vs. grupo 7 (dividir el tamaño del grupo 6 entre el tamaño del grupo 7). En nuestro trabajo fue de 0.7; generalmente es de 2:1 debido a que la presentación de nalgas es más frecuente en nulíparas que en multíparas. Si la proporción es diferente, sospeche una proporción inusual nulípara/multípara o inadecuada recolección de datos.

\section{Pasos para evaluar las tasas de cesáreas}

- El tamaño de grupo 9 (columna 2) está dentro del rango esperado, 1.1\%; sin embargo, la tasa de cesárea del $100 \%$ indica que por las normas del servicio en todo producto en mala posición debe interrumpirse el embarazo por vía abdominal en cuanto se 
diagnostique si la edad gestacional y estado del trabajo de parto lo indica.

- El tamaño de los grupos 1+2 (columna 3) es el 37.9\%, el cual está dentro del rango esperado. La tasa del tamaño del grupo 1/grupo 2 es 7.2, la cual está dentro de lo esperado (> 2.1) e indica que el hospital no practica muchas inducciones o cesáreas antes del trabajo de parto en nulíparas.

- El tamaño de los grupos 3+4 (columna 3) es el 26.5\%, esto sugiere que el hospital proporciona servicio a una población con alto grado de fertilidad. La tasa de cesárea del grupo 3/grupo 4 es 14.5, lo cual es lo esperado (es más alto que la tasa del grupo 1/grupo 2) e indica que en ese hospital no realizan muchas inducciones o cesáreas en multíparas sin cicatrices de cesáreas previas.

- El tamaño de grupo 5 (columna 3) es el 19.3\%, el cual es relativamente alto e indica que este hospital recibe muchas mujeres con cesáreas previas, probablemente aquellas que tuvieron su primera cesárea en ese hospital.

- El tamaño de los grupos 6+7 (columna 3) es el $2.7 \%$, el cual está dentro de lo esperado para partos en nalgas. La tasa de grupo 6/grupo 7 fue 0.68, lo cual es raro, ya que los partos de nalgas son más frecuentes en nulíparas que en multíparas. Esto podría indicar inadecuada colección de datos y sería importante revisar los registros de esos grupos.

- El tamaño del grupo 8 (columna 3) fue el 1.1\%, lo cual está cerca a la prevalencia esperada de embarazos múltiples en la población general.

- El tamaño del grupo 10 (columna 3) fue el 11.5\%, el cual es alto e indica que el hospital está recibiendo pacientes de alto riesgo de otros hospitales locales.

Tasas de cesárea por grupos y contribución.

- La tasa de cesárea para el grupo 1 (columna 5) es el $32.4 \%$, el cual es alto, comparado al promedio de otros hospitales; lo ideal debe ser un 10\%. Sería interesante revisar las indicaciones promedio de la cesárea en este grupo y los protocolos clínicos sobre el manejo del parto para nulíparas en trabajo de parto espontáneo con producto único en presentación cefálica a término.
- La tasa de cesárea para el grupo 2 (columna 5) es el 88.6\%; es muy alta, comparada con el promedio de otros hospitales (35-40\%).

- La tasa de cesárea para el grupo 3 (columna 5) es el $13.1 \%$, está cerca a lo esperado e indica que el manejo de multíparas en trabajo de parto espontáneo a término es adecuado.

- La tasa de cesárea para el grupo 4 (columna 5) es el $78.1 \%$, lo cual es mucho más alto que el promedio $(<20 \%)$.

- La tasa de cesárea para el grupo 5 (columna 5) es el $79 \%$, el cual es muy alto. Esto indica que hay pocos protocolos de atención de parto después de cesárea.

- La tasa de cesárea para el grupo 8 (columna 5) es el 95\%, el cual es más alto que el promedio, del 60\%. Podría implementarse una política más liberal hacia permitir el parto en embarazos gemelares o en la población de alto riesgo.

- La tasa de cesárea del grupo 10 (columna 5) es del $65.4 \%$, la cual es alta y sugiere que la mayoría de las mujeres que presentan parto pretérmino en este hospital probablemente no inicien con trabajo de parto espontáneamente y se les hayan practicado cesárea antes del trabajo de parto debido a complicaciones como preeclampsia o restricción en el crecimiento intrauterino.

- El único grupo que más contribuyó a la tasa global de cesárea fue el grupo 5, con cerca de un tercio de los casos (31.8\%), columna 6. Por lo tanto, tiene sentido implementar intervenciones para reducir la tasa de cesárea en este grupo. Podría iniciar con auditarse a todas las mujeres con cesárea previa y a cuántas de ellas se les ofrece tener un parto después de la cesárea ${ }^{15,16}$.

\section{DISCUSIÓN}

En el presente estudio se analizó la tasa de cesáreas en un periodo de cinco años, donde se observa una disminución significativa, alcanzando la tasa más alta en el año 2014 (50\%) con una disminución progresiva en años posteriores, alcanzando en el último año una tasa del $43.7 \%$. Sin embargo, continúa siendo superior 
a la recomendada por la OMS, del $15 \%$. Dentro de la clasificación de Robson el grupo más frecuente fue el grupo 1; sin embargo, el grupo que más aportó a la tasa de cesáreas fue el grupo 5, multigestas con embarazo de 37 semanas y con cicatriz uterina previa, donde la indicación más frecuente fue antecedente de 2 cesáreas previas. Dentro de este grupo podemos observar que a casi el 100\% de las pacientes con 2 cesáreas previas o más se les realiza cesárea, ya que se trata de una indicación absoluta para su realización, pero es importante mencionar el grupo de mujeres que solo presentan una cesárea previa, a las que al $67 \%$ se les realizó cesárea, asumiendo que el tener el antecedente de una cesárea previa la predispone a tener una nueva cesárea en cerca del $70 \%$. Por ello se evidencia la importancia de prevenir la primera cesárea.

El grupo que ocupa el segundo lugar en aporte a la tasa de cesáreas es el grupo de primigestas con embarazo de término, trabajo de parto espontáneo, presentación cefálica y que, además, es el grupo de Robson más frecuente en nuestra población; se observó un porcentaje de cesáreas del 34\%, siendo la principal indicación la falta de progresión de trabajo de parto. Vale la pena analizar y revisar estrictamente las definiciones y el protocolo sobre la progresión del trabajo de parto.

Otro grupo importante fue el de pacientes con embarazos menores de 37 semanas (grupo 10) a las que al $65 \%$ se les realizó cesárea, siendo la indicación principal el trabajo de parto pretérmino.

Los grupos 6, 7, 8 y 9 son pacientes con embarazo con presentación pélvica, embarazos múltiples y situación transversa; tuvieron un porcentaje de cesáreas del $100 \%$. Cabe mencionar que este porcentaje tan elevado es debido a que, dentro de los protocolos de nuestra institución, la vía de resolución del embarazo es abdominal y no se ponen en práctica maniobras de versión externa o parto instrumentado.

\section{CONCLUSIONES}

Se concluye que en nuestra población atendida en el Hospital Juárez de México el grupo que más aporta a la tasa de cesáreas es el grupo 5, mujeres con cicatrices uterinas previas, similar a lo publicado por
Brennan, et al. ${ }^{17}$; el segundo grupo que más aporta a la tasa de cesáreas es el grupo 1, que también es el más frecuente en nuestra población. La problemática de una tasa de cesáreas por arriba de lo recomendado por la OMS continúa siendo un reto para la mayoría de las instituciones de salud; sin embargo, esta tasa de cesáreas es dependiente directamente de las características de la población atendida y del tipo de institución, ya que el hecho de ser institución escuela influye ampliamente en la tasa elevada de cesáreas. Así mismo, por tratarse de un hospital general de alta especialidad, hay un gran porcentaje de pacientes atendidas con patologías agregadas graves que favorecen la resolución del embarazo por cesárea.

Deben analizarse detalladamente los protocolos de atención, individualizando a cada paciente de acuerdo a sus características y necesidades. Debemos ajustarnos a las recomendaciones normativas. Se concluye también, al igual que en otros estudios reportados, que uno de los puntos clave para reducir la tasa de cesáreas es evitando la primera cesárea en las pacientes, siempre y cuando no se vea afectado el bienestar maternofetal. Se propone realizar un estudio aún más detallado para reducir la tasa de cesáreas, analizando de manera individual las cesáreas realizadas por cada miembro del personal encargado del área de ginecoobstetricia, y así mismo un análisis detallado de las principales indicaciones de la primera cesárea. Es importante capacitar al personal a cargo del servicio de Obstetricia en todos los turnos sobre monitorización del bienestar fetal, así como establecer protocolos de manejo de los principales diagnósticos de las indicaciones de cesáreas.

\section{BIBLIOGRAFÍA}

1. Betran AP, Torloni MR, Zhang JJ, Gülmezoglu AM; WHO Working Group on Caesarean Section. WHO Statement on Caesarean Section Rates. BJOG. 2016;123(5):667-70.

2. Sexual and reproductive health [Internet]. Ginebra: Organización Mundial de la Salud; 2019. Disponible en: www.who.int/reproductivehealth/

3. Ye J, Betran AP, Guerrero Vela M, Souza JP, Zhang J. Searching for the optimal rate of medically necessary cesarean delivery. Birth. 2014;41(3): 237-44.

4. Armstrong JC, Kozhimannil KB, McDermott P, Saade GR, Srinivas SK Society for Maternal-Fetal Medicine Health Policy Committee. Comparing variation in hospital rates of cesarean delivery among low-risk women using 3 different measures. Am J Obstet Gynecol. 2016;214(2): 153-63.

5. Spong CY, Berghella V, Wenstrom KD, Mercer BM, Saade GR. Preventing the first cesarean delivery: summary of a joint Eunice Kennedy Shriver National Institute of Child Health and Human Development, Society for Maternal-Fetal Medicine, and American College of Obstetricians and Gynecologists workshop. Obstet Gynecol. 2012;120:1181-93. 
6. American College of Obstetrics and Gynecology; Society for Maternal-Fetal Medicine. Safe prevention of the primary cesarean delivery. Obstetric care consensus no. 1. Obstet Gynecol. 2014;123:693-711.

7. Clark SL, Belfort MA, Dildy GA, Herbst MA, Meyers JA, Hankins GD. Maternal death in the 21st century: causes, prevention and relationship to cesarean delivery. Am J Obstet Gynecol. 2008;199:36.e1-5.

8. Tapia V, Betran AP, Gonzales GF. Caesarean section in Peru: Analysis of trends using the Robson classification system. PLoS One. 2016; 11(2): $\mathrm{e} 0148138$

9. Jayot A, Nizard J. Evolution of cesarean categories in a modified Robson classification in a single center from 2002 to 2012 due to high rate of maternal pathology. J Obstet Gynaecol Res. 2016;42(6):648-54.

10. Robson M, Murphy M, Byrne F. Quality assurance: The 10-Group Classification System (Robson classification), induction of labor, and cesarean delivery. Int J Gynaecol Obstet. 2015:131(Suppl 1):S23-7.

11. Lamy C, Alexander S. [The World Health Organization proposes adopting the Robson Classification as an internationally applicable cesarean section classification system]. J Gynecol Obstet Biol Reprod (Paris). 2015:44(7):587-90.

12. Torloni MR, Betran AP, Souza JP, Widmer M, Allen T, Gulmezoglu M, et al. Classifications for cesarean section: a systematic review. PLOS One. 2011;6(1):e14566
13. Vogel JP, Betran AP, Vindevoghel N, Souza JP, Torloni MR, Zhang J, et al.; WHO Multi-Country Survey on Maternal and Newborn Health Research Network. Use of the Robson classification to assess caesarean section trends in 21 countries: a secondary analysis of two WHO multicounty surveys. Lancet Glob Health. 2015;3(5):e260-70.

14. Manny-Zitle Al, Tovar-Rodríguez JM. Incidencia de la operación cesárea según la clasificación de Robson en el Servicio de Ginecología y Obstetricia del hospital general Dr. Fernando Quiroz Gutierrez. Instituto de Seguridad Social al Servicio de los Trabajadores del Estado. Cir Cir. 2018; 86:1-9.

15. Robson Classification: Implementation Manual [Internet]. Ginebra: Organización Mundial de la Salud; 2017. Disponible en: www.who.int/reproductivehealth/publications/maternal_perinatal_health/robson-classification/en

16. WHO | The Robson classification implementation manual https://www.who. int/reproductivehealth/topics/maternal_perinatal/robson-classification-implementati

17. Brennan D, Robson M, Murphy M, O'Herlihy C. Comparative analysis of international cesarean delivery rates using 10-group classification identifies significant variation in spontaneous labor. Am J Obstet Gynecol. 2009:201:308.e1e8. 